Original

\title{
Assessment of quality and interpretation of panoramic radiographs obtained in the Lao People's Democratic Republic as part of a teleradiology collaboration with Japan
}

\author{
Kunihito Matsumoto1,2), Souksavanh Vongsa3), Ichiro Nakajima ${ }^{4,5)}$, Ken-ichiro Ejima ${ }^{1,2)}$, \\ Kiyomi Kohinata1), Toshihiro Suzuki'1), Shigeharu Hosono6), Hirofumi Aboshi ${ }^{7,8)}$, \\ Fumiyuki Kuwata ${ }^{5,9)}$, and Kichibee Otsuka ${ }^{10}$
}

\author{
1)Department of Oral and Maxillofacial Radiology, Nihon University School of Dentistry, Tokyo, Japan \\ 2)Division of Advanced Dental Treatment, Dental Research Center, Nihon University School of Dentistry, \\ Tokyo, Japan \\ 3)Faculty of Dentistry, University of Health Sciences, Vientiane, Lao People's Democratic Republic \\ 4)Department of Community Dentistry, Nihon University School of Dentistry, Tokyo, Japan \\ ${ }^{5)}$ Division of Dental Education, Dental Research Center, Nihon University School of Dentistry, Tokyo, Japan \\ 6)Department of Pediatrics, Nihon University School of Medicine, Tokyo, Japan \\ 7)Department of Legal Medicine, Nihon University School of Dentistry, Tokyo, Japan \\ ${ }^{8)}$ Division of Social Dentistry, Dental Research Center, Nihon University School of Dentistry, Tokyo, Japan \\ 9)Department of Chemistry, Nihon University School of Dentistry, Tokyo, Japan \\ ${ }^{10)}$ Department of Biochemistry, Nihon University School of Dentistry, Tokyo, Japan
}

(Received March 3, 2015; Accepted May 21, 2015)

\begin{abstract}
As part of quality assessment of a teleradiology program we evaluated the validity of patient information received, the quality of panoramic radiography imaging in Laos, and the ability of a Laotian radiologist to detect temporomandibular joint abnormalities. The amount of patient information gathered from 2,021 scans of panoramic radiographs was evaluated by triage before image diagnosis. Among the radiographs from 2,021 patients, primary triage indicated that there was insufficient information for $794(39.3 \%)$ patients. Secondary triage to assess imaging failure included 1,227 radiographs, four of which were excluded from imaging diagnosis because
\end{abstract}

Correspondence to Dr. Kunihito Matsumoto, Department of Oral and Maxillofacial Radiology, Nihon University School of Dentistry, 1-8-13 Kanda-Surugadai, Chiyoda-ku, Tokyo 101-8310, Japan

Fax: +81-3-3219-8354

E-mail: matsumoto.kunihito@nihon-u.ac.jp

doi.org/10.2334/josnusd.57.235

DN/JST.JSTAGE/josnusd/57.235 of unacceptable image flaws. In total, 2,446 joints from 1,223 radiographs were evaluated for temporomandibular joint abnormalities in order to compare the image interpretation abilities of Laotian and Japanese radiologists. The kappa coefficient was $0.836(P<$ 0.01 ) for the agreement between the two observers in detecting temporomandibular joint abnormalities on radiographs. We conclude that additional efforts are needed in order to overcome the challenges of maintaining quality in imaging techniques and diagnoses in Laos. (J Oral Sci 57, 235-239, 2015)

Keywords: teleradiology; panoramic radiography; quality assessment; temporomandibular joint.

\section{Introduction}

A research project utilizing teleradiology was started between the University of Health Sciences (UHS) of the Lao People's Democratic Republic (Lao PDR) and the 
Nihon University School of Dentistry in Japan. The Lao PDR remains one of the poorest countries in Southeast Asia. Despite the rapid growth of its economy, with a gross domestic product growth rate of $8.3 \%$ per year, the Lao PDR ranks 138th on the Human Development Index, indicating that it has lower median development than those of neighboring countries (The World Factbook 2013-14). However, the rate of annual improvement on the Human Development Index during 1990-2012 was 1.53 , and recent improvement in the nutritional status of children under age 5 years indicates that economic growth is having an effect and that medical and environmental indices have improved (1). Data on the dental health of Laotians are limited, and most reports have focused on children (2-6). The evidence indicates that the prevalence of dental caries differs between Laotian children living in urban and rural areas $(2,3)$, while the incidence of gingivitis is high in all settings $(2,3)$. Motohashi et al. (3) reported that Laotian children who live in rural areas showed no signs of temporomandibular disorders in clinical evaluations. In general, temporomandibular disorders vary by region but are limited to adult populations in Southeast Asian countries.

Teleradiology is the field of telemedicine that involves electronic transmission of diagnostic images from one location to another for interpretation and/or consultation regarding diagnosis and/or treatment decision-making (7). Teleradiology includes inter-hospital, inter-regional, and international applications and has a number of roles. As in the present study, international use of teleradiology between developing and developed countries may improve health technology, including on-site systems, and assist in clinical diagnosis and treatment. We conducted the present study to maintain quality assurance as part of our frequent quality assessment of teleradiology.

Panoramic radiography (PR) is used worldwide for the screening of oral and maxillofacial diseases such as periodontitis, dental caries, inflammatory diseases, tumorous lesions, and osteoarthritis of the temporomandibular joint (TMJ). The first PR apparatus in the Lao PDR was installed in the UHS dental hospital. Considerable expertise and experience is necessary when reading panoramic radiographs to screen for lesions and abnormalities. Few oral and maxillofacial radiologists and radiation technologists work in the Lao PDR. In addition, specialized training programs for oral and maxillofacial radiology (OMFR) specialists are not available in the country. Thus, assessment and diagnosis using radiographs are uncommon in the Lao PDR.

As part of a quality assessment of our teleradiology collaboration, we evaluated 1) the validity of patient information gathered during teleradiology, 2) the PR imaging technique at the Lao PDR UHS, and 3) the ability of Laotian oral and maxillofacial radiologists (OMF radiologists) to interpret images and detect TMJ abnormalities.

\section{Materials and Methods \\ Study subjects}

The study subjects were 2,021 patients who underwent PR for assessment of periodontitis, dental caries, inflammatory disease, and/or temporomandibular disorders at the UHS Faculty of Dentistry between April 2012 and August 2014. Patients were excluded if they had large tumorous or cystic lesions, mandibular trauma, or suspected systemic disease, as facial asymmetry, expansion, or swelling could prevent proper positioning during scanning.

The use of the clinical data in this study was reviewed and approved by the bioethics committee of the UHS Faculty of Dentistry (No. 010/14), and the study was performed in accordance with the ethical standards established in the 2013 revision of the Declaration of Helsinki. All patients provided written informed consent to participate after receiving a detailed explanation of the study purposes.

\section{PR imaging}

A Veraviewepocs CCD digital device (J. Morita Corp., Kyoto, Japan) at the UHS Faculty of Dentistry was used for image acquisition. Multiple dentists performed PR scanning, using reference planes such as the Frankfort horizontal plane and midsagittal plane. The exposure settings were set to automatic. All radiographs were formatted as digital imaging and communications in medicine (DICOM) files and were stored on the image server at the UHS Faculty of Dentistry.

\section{Teleradiology system}

The ViewSend Medical system (ViewSend ICT Co., Ltd., Tokyo, Japan) was used to export the DICOM-formatted PR imaging data from the UHS Faculty of Dentistry to the Nihon University School of Dentistry Department of OMFR.

\section{Triage of patient information based on DICOM format (primary triage)}

PR DICOM datasets sent to Nihon University were first triaged for patient information (textual DICOM information). Datasets that lacked basic patient information, such as age, sex, or inspection date (the minimum information required to identify both patient and examination type), 
Table 1 Triage of patient information (textual DICOM information)

\begin{tabular}{lc}
\hline Primary triage & No. $(\%)$ \\
\hline Sufficient information & $1,227(60.7 \%)$ \\
Insufficient information & $794(39.3 \%)$ \\
Total & $2,021(100 \%)$ \\
\hline
\end{tabular}

DICOM: digital imaging and communications in medicine Among the 2,021 panoramic radiography datasets, 794 had insufficient patient information on patient and/or examination type.

Table 2 Triage of imaging failure

\begin{tabular}{lc}
\hline Secondary triage & No. $(\%)$ \\
\hline Poor & $4(0.3 \%)$ \\
Fair & $8(0.7 \%)$ \\
Good & $97(7.9 \%)$ \\
Excellent & $1,118(91.1 \%)$ \\
Total & $1,227(100 \%)$ \\
\hline
\end{tabular}

Four panoramic radiographs with serious flaws (poor) were excluded from image evaluation. Those classified as excellent had no image flaws.

were excluded from the triage on the basis of imaging failure. This triage was performed by one Japanese OMFR specialist (KM), who is certified by the Japanese Society of OMFR.

\section{Triage of imaging failure (secondary triage)}

To screen for maxillar and mandibular abnormalities, radiographs without missing patient information were evaluated for the presence or absence of image flaws. All radiographs were classified according to the following criteria: poor - unacceptable for diagnosis because of a serious flaw, fair-acceptable with a moderate flaw, good-acceptable with a minor flaw, and excellent-no image flaws.

The type of failure was recorded, and radiographs with serious flaws (poor) were excluded from diagnostic image evaluation. This triage was also performed by one Japanese OMFR specialist.

\section{Evaluation of TMJ bone abnormalities}

One Laotian OMF radiologist (SV) and one Japanese OMFR specialist (KM) separately examined all radiographs, excluding those with both missing patient information and serious flaws on the PR. The presence/ absence of bone abnormalities of the TMJ condyle was
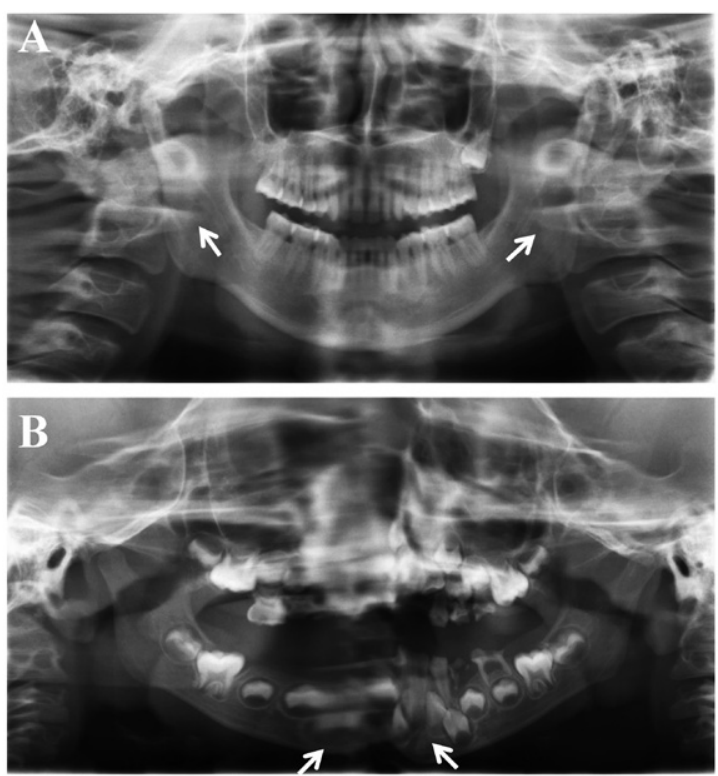

Fig. 1 Examples of panoramic radiographs excluded in secondary triage.

A: Horizontal minification of anterior teeth and ghost images of vertebrae overlapping with the ramus (arrows) because of marked failure in anteroposterior positioning of the setting of the focal trough. B: Artifact in the anterior region resulting from patient movement during imaging (arrows), and a bilateral difference in magnification because of a failure in head-setting.

evaluated on both sides of the TMJ, using the modified Uemura criteria (8). The joint was classified as abnormal when an observer noted eburnation, sclerosis, erosion, concavity, deformity, extensive flattening, marginal proliferation, or a calcified condyle body.

\section{Statistical analysis}

Using SPSS 17 software (IBM Japan, Tokyo, Japan), we calculated the kappa coefficient in order to evaluate interobserver agreement between the Laotian and Japanese radiologists. The kappa coefficient was interpreted based on the criteria of Landis and Koch (9). A $P$ value of less than 0.05 was considered to indicate statistical significance.

\section{Results}

In primary triage, the reviewer found that patient information was insufficient for 794 (39.3\%) of the 2,021 cases (Table 1). Secondary triage (of imaging failure) included 1,227 radiographs, and 109 (8.9\%) had an image flaw. These flawed radiographs were classified as poor, fair, or good in 4, 8, and 97 cases, respectively (Table 2). Examples of poor radiographs are shown in Fig. 1. The most frequent flaws in radiographs were ghost images of piercings, hairpins, necklaces, and/or dentures (Table 3). Four 
Table 3 Characteristics of flaws in panoramic radiographs

\begin{tabular}{lc}
\hline Type of flaw or imaging error & No. $(\%)$ \\
\hline Ghost image of piercings, hairpins, necklaces, and/or dentures & $67(60.4 \%)$ \\
Failure of head setting (Frankfort horizontal plane positioning) & $18(16.2 \%)$ \\
Failure of focal trough setting (anteroposterior positioning) & $23(20.7 \%)$ \\
Movement during imaging & $3(2.7 \%)$ \\
Total & $111(100 \%)$ \\
\hline
\end{tabular}

A few radiographs had multiple flaws.

Table 4 Characteristics of patients

\begin{tabular}{lrcc}
\hline Sex & No. & Mean age & Range \\
\hline Male & 596 & 36.0 & $3-88$ \\
Female & 627 & 30.5 & $5-87$ \\
Total & 1,223 & 33.2 & $3-88$ \\
\hline
\end{tabular}

poor radiographs were excluded in the TMJ evaluation. There were no image flaws in 1,118 radiographs $(91.1 \%)$, which were classified as excellent. In total, 2,446 joints in 1,223 radiographs were evaluated for TMJ abnormalities by the two observers. The characteristics of the patient are shown in Table 4. The Laotian and Japanese OMFR specialists identified TMJ abnormalities in 72 and 99 joints, respectively (Table 5). The kappa coefficient was 0.836 for the comparison of the judgments of the two observers in detecting TMJ abnormalities on radiographs $(P<0.01)$, which was considered very good agreement.

\section{Discussion}

Our study found that $39.9 \%$ of the files lacked one or more pieces of textual DICOM information needed to identify a patient or examination (DICOM data consists of textual and image information) (10). Teleradiology is generally performed using DICOM standards, and the absence of this important identifying information is a major concern in our teleradiology system. This information might have been missing because of limitations in the national health administration of the Lao PDR. Furthermore, the fact that the Lao PDR has only one dental hospital means that only a limited number of dentists and co-dental staff are available to treat all dental patients. This situation is an important issue in developing countries seeking to improve national health and requires medium- and longterm international financial and development assistance, and human resource development, from developed countries to resolve these issues.

Evaluations of imaging failure showed that most PR imaging flaws were minor failures, i.e., the images were
Table 5 Comparison between observers of detectability of temporomandibular joint bone abnormalities

\begin{tabular}{cccrc}
\hline & & \multicolumn{3}{c}{ Observer 2(Japan) } \\
& & $(+)$ & $(-)$ & Total \\
\hline \multirow{2}{*}{ Observer 1 } & $(+)$ & 71 & 1 & 72 \\
(Lao PDR) & $(-)$ & 28 & 2,346 & 2,374 \\
& Total & 99 & 2,347 & 2,446 \\
\hline
\end{tabular}

PDR: People's Democratic Republic

still acceptable for screening of jaw abnormalities. Some Laotian dentists received instruction on PR techniques from Japanese dentists and technicians when the PR apparatus was installed in the UHS and subsequently taught the techniques to other dentists at the hospital. Our study included all radiographs obtained by the Laotian dentists, regardless of whether they received direct or indirect instruction in the technique. The results indicate that all the Laotian dentists adequately performed PR imaging. The good technique of the dentists receiving indirect instruction suggests that the educational system at UHS is sufficient. Consequently, examinations of technical proficiency for modalities such as intraoral and cephalography imaging should be performed in future studies.

The present study examined the detectability of TMJ abnormalities on radiographs in order to evaluate the validity of image interpretations as a reference index. Initially, we considered other diseases as reference indices for image interpretation, including tumorous and cystic lesions and inflammation, including marginal periodontitis. However, there were few cases of tumorous and cystic lesions and many cases of inflammatory disease. In addition, large tumorous lesions could influence positioning for imaging; thus, patients with these conditions were excluded from the study. Since PR imaging was established, it has been widely used for evaluating and screening TMJ abnormalities. The present results were much better than those of previous studies that used radiographs to evaluate interobserver agreement in TMJ abnormality assessment, including those of Dahlström and Lindvall (11) and Ahmad et al. (12), perhaps because 
of differences in the criteria used and observer calibration. In the present study, we did not evaluate a particular type of abnormality but instead evaluated the presence/ absence of TMJ abnormalities. Regarding observer calibration, the Japanese and Laotian observers have used the teleradiology system for several years to perform consensual decision-making in clinical image diagnosis of patients in the Lao PDR. This collaboration between the two countries explains the excellent calibration level between observers and the high level of agreement. Thus, the ability of the Laotian OMF radiologists to diagnose TMJ abnormalities was similar to that of the Japanese OMFR specialists. In a future study, we will further assess the interpretation abilities of Laotian OMF radiologists by examining the detectability of other diseases on radiographs.

This study was the first to examine the validity of patient information during teleradiology, PR imaging technique, and the diagnostic abilities of Laotian OMF radiologists. However, this study did have limitations, namely, intraoral radiography was performed more frequently than necessary and more observers are needed in the Lao PDR and Japan in order to evaluate and compare the ability to interpret images between countries.

This study evaluated the quality of 1) information gathered for use in teleradiology, 2) PR imaging, and 3) interpretation of TMJ bone abnormality screening using a teleradiology system in the Lao PDR. We found that patient information in the DICOM format was not appropriately managed in $39.3 \%$ of the images, which was identified as a major issue in the Lao PDR. However, PR imaging and the ability to interpret these images for TMJ bone abnormalities were equivalent in Laotian and Japanese OMF radiologists.

\section{Acknowledgments}

This study was supported by the Nihon University President Grant Initiative (2012-2014), the Sato Fund (2014), and a grant from the Nihon University School of Dentistry Dental Research Center (2014).

\section{References}

1. United Nations Development Programme (2013) Human development report 2013. The rise of the South: human progress in a diverse world. UNDP, New York, 143-151.

2. Chuckpaiwong S, Ngonephady S, Dharmbhibhit J, Kasetsuwan J, Sirirat M (2000) The prevalence of periodontal disease and oral hygiene care in Savannakhet Province, Lao People's Democratic Republic. Southeast Asian J Trop Med Public Health 31, 775-779.

3. Motohashi M, Nakajima I, Aboshi H, Honda K, Yanagisawa M, Miyata T et al. (2009) The oral health of children in a rural area of the Lao People's Democratic Republic. J Oral Sci 51, 131-135.

4. Jürgensen N, Petersen PE (2009) Oral health and the impact of socio-behavioural factors in a cross sectional survey of 12-year old school children in Laos. BMC Oral Health 9, 29.

5. Besseling S, Ngonephady S, van Wijk AJ (2013) Pilot survey on dental health in 5-12-year-old school children in Laos. J Investig Clin Dent 4, 44-48.

6. Sisounthone J, Ejima K, Nakajima I, Honda K, Hosono S, Vongsa $\mathrm{S}$ et al. (2015) Application of telemedicine to assess mandibular cortical width on panoramic images of dental patients in the Lao People's Democratic Republic. Oral Radiol. (in press)

7. Martí-Bonmatí L, Morales A, Donoso Bach L (2012) Toward the appropriate use of teleradiology. Radiologia 54, 115-123.

8. Matsumoto K, Honda K (2013) Temporomandibular disorders and morphology of temporomandibular joints. Practice in Prosthodontics, Extra Iss, 69-75.

9. Landis JR, Koch GG (1977) The measurement of observer agreement for categorical data. Biometrics 33, 159-174.

10. Kahn CE Jr, Langlotz CP, Channin DS, Rubin DL (2011) Informatics in radiology: an information model of the DICOM standard. Radiographics 31, 295-304.

11. Dahlström L, Lindvall AM (1996) Assessment of temporomandibular joint disease by panoramic radiography: reliability and validity in relation to tomography. Dentomaxillofac Radiol 25, 197-201.

12. Ahmad M, Hollender L, Anderson Q, Kartha K, Ohrbach R, Truelove EL et al. (2009) Research diagnostic criteria for temporomandibular disorders (RDC/TMD): development of image analysis criteria and examiner reliability for image analysis. Oral Surg Oral Med Oral Pathol Oral Radiol Endod $107,844-860$. 